\title{
Omega-3 Polyunsaturated Fatty Acids Enriched Hen Eggs Consumption Enhances Microvascular Reactivity in Young Healthy Individuals
}

\begin{tabular}{|c|c|}
\hline Journal: & Applied Physiology, Nutrition, and Metabolism \\
\hline Manuscript ID & apnm-2017-0735.R2 \\
\hline Manuscript Type: & Article \\
\hline Date Submitted by the Author: & 07-Mar-2018 \\
\hline Complete List of Authors: & $\begin{array}{l}\text { Stupin, Ana; Faculty of Medicine J.J. Strossmayer University of Osijek, } \\
\text { Department of Physiology and Immunology } \\
\text { Rasic, Lidija; Faculty of Medicine J.J. Strossmayer University of Osijek, } \\
\text { Department of Physiology and Immunology } \\
\text { Matic, Anita; Faculty of Medicine J.J. Strossmayer University of Osijek, } \\
\text { Department of Physiology and Immunology } \\
\text { Stupin, Marko; Faculty of Medicine J.J. Strossmayer University of Osijek, } \\
\text { Department of Physiology and Immunology; Klinicki bolnicki centar Osijek, } \\
\text { Department for Cardiovascular Disease } \\
\text { Kralik, Zlata; Faculty of Agriculture, Josip Juraj Strossmayer University of } \\
\text { Osijek, Department for Special Zootechnics } \\
\text { Kralik, Gordana; Faculty of Agriculture, Josip Juraj Strossmayer University } \\
\text { of Osijek, Department for Special Zootechnics } \\
\text { Grcevic, Manuela; Faculty of Agriculture, Josip Juraj Strossmayer } \\
\text { University of Osijek, Department for Special Zootechnics } \\
\text { Drenjancevic, Ines; Faculty of Medicine J.J. Strossmayer University of } \\
\text { Osijek, Department of Physiology and Immunology }\end{array}$ \\
\hline Keyword: & $\begin{array}{l}\text { omega-3 PUFAs, vascular function, microcirculation, laser Doppler } \\
\text { flowmetry, serum lipid profile }\end{array}$ \\
\hline $\begin{array}{r}\text { Is the invited manuscript for } \\
\text { consideration in a Special } \\
\text { Issue? : }\end{array}$ & $\mathrm{N} / \mathrm{A}$ \\
\hline
\end{tabular}




\title{
Omega-3 Polyunsaturated Fatty Acids Enriched Hen Eggs Consumption Enhances Microvascular Reactivity in Young Healthy Individuals
}

\author{
Ana Stupin $^{\mathrm{a}^{*}}$, Lidija Rasic ${ }^{\mathrm{a}^{*}}$, Anita Matic ${ }^{\mathrm{a}}$, Marko Stupin ${ }^{\mathrm{a}, \mathrm{b}}$, Zlata Kralik ${ }^{\mathrm{c}}$, Gordana Kralik ${ }^{\mathrm{c}}$, \\ Manuela Grcevic ${ }^{\mathrm{c}}$, Ines Drenjancevic ${ }^{\mathrm{a} \dagger}$
}

${ }^{a}$ Department of Physiology and Immunology, Faculty of Medicine, Josip Juraj Strossmayer

University of Osijek, Osijek, Croatia

${ }^{\mathrm{b}}$ Department for Cardiovascular Disease, Osijek University Hospital, Osijek, Croatia

${ }^{\mathrm{c}}$ Department for Special Zootechnics, Faculty of Agriculture, Josip Juraj Strossmayer University of Osijek, Osijek, Croatia

Author's contributions: *Ana Stupin and Lidija Rasic equally contributed to this paper.

\section{${ }^{\dagger}$ Corresponding author:}

Ines Drenjancevic, $\mathrm{MD}, \mathrm{PhD}$

Department of Physiology and Immunology

Faculty of Medicine, Josip Juraj Strossmayer University of Osijek

Cara Hadrijana 10E, HR-31 000, Osijek, Croatia

Phone: +38531512800

Fax: +38531512866

Email: ines.drenjancevic@mefos.hr

2

\section{Author's e-mail addresses:}

Ana Stupin - cavka.ana@gmail.com; Lidija Rasic - rasiclid@gmail.com; Anita Matic -

5 anita.cosic@mefos.hr; Marko Stupin - marko.stupin@gmail.com; Zlata Kralik -

6 zlata.kralik@pfos.hr; Gordana Kralik - Gordana.Kralik@pfos.hr; Manuela Grcevic -

7 mgrcevic@pfos.hr 


\section{Abstract}

Whilst the beneficial effect of omega-3 polyunsaturated fatty acids (PUFAs) supplementation on cardiovascular (CV) system is well supported in $\mathrm{CV}$ patients, the effect of consumption of omega-3 PUFAs enriched functional food in healthy individuals is still not fully elucidated. This study aimed to determine the effect of consumption of omega-3 PUFAs enriched hen eggs on microvascular reactivity (primary outcome), blood pressure (BP) and serum lipid profile in young healthy individuals. Control group $(\mathrm{N}=16)$ ate three ordinary hen eggs $(277 \mathrm{mg}$ omega-3 PUFAs/day), and OMEGA-3 group $(\mathrm{N}=20)$ ate three omega-3 PUFAs enriched eggs containing 259 mg of omega-3 PUFAs/egg daily (ALA 167 mg/egg, EPA 7 mg/egg, DHA 84 mg/egg) for 3 weeks (777 mg omega-3 PUFAs/day). Post-occlusive reactive hyperemia (PORH) in skin microcirculation assessed by laser Doppler flowmetry, serum lipid profile, fasting blood glucose, high-sensitivity C-reactive protein (hsCRP) and arterial BP were measured in all subjects before and after the protocol. PORH was significantly enhanced, and triglycerides, hsCRP and BP were significantly decreased in OMEGA-3 group compared to baseline measurement, while there was no significant difference in Control group after the protocol compared to baseline. This is the first study to demonstrate that consumption of a mixture of omega-3 PUFAs (ALA+EPA+DHA), provided via enriched hen eggs, elicits changes in microvascular reactivity, BP and triglycerides level in healthy subjects that are associated with CV benefits, thus suggesting that daily consumption of omega-3 PUFAs enriched eggs in healthy individuals may potentially contribute to $\mathrm{CV}$ risk factors attenuation and disease prevention.

Key words: omega-3 PUFAs, vascular function, microcirculation, laser Doppler flowmetry, serum lipid profile 


\section{2}

\section{Introduction}

Nowadays, it is widely accepted that an impaired vascular endothelial function represents one of the main features of cardiovascular (CV) diseases (Ross 1999). Consumption of omega-3 polyunsaturated fatty acids (PUFAs) has been shown to reduce CV risk and has a beneficial effect on CV disease progression (Auger et al. 2016). It has been widely investigated whether intake of omega-3 PUFAs may prevent or delay the progression of atherosclerosis via its impact on the initial steps in its pathogenesis, i.e. changes in endothelial and vascular function (Endo and Arita 2016), as opposed to "traditional" CV risk factors such as blood pressure (BP), triglyceride concentrations and/or inflammation. Considerable knowledge on this issue was provided by studies in animal model (Gortan Cappellari et al. 2013; Lopez et al. 2004; Zhang et al. 2013), which demonstrated that omega-3 PUFAs have the potential to improve vascular and endothelial function by: a) increasing bioavailability of nitric oxide (NO) (Gortan Cappellari et al. 2013; Lopez et al. 2004; Zhang et al. 2013); b) reducing oxidative stress level (Gortan Cappellari et al. 2013; Zhang et al. 2013); and/or c) attenuating inflammation (Wang et al. 2011). In order to test knowledge obtained in animal studies, a number of human functional vascular studies were performed that mainly investigated the effect of omega-3 PUFAs (in the form of eicosapentaenoic acid (EPA) + docosahexaenoic acid (DHA) capsules or walnut-supplemented diet rich in $\alpha$-linolenic acid (ALA)) on the reactivity of large (conductance) arteries using flow mediated dilation (FMD) of the brachial artery (Siasos et al. 2013; Rizza et al. 2009; Shah et al. 2007). There is a paucity of data on the effect of omega-3 PUFAs supplementation on microvascular reactivity which is usually non-invasively tested by the laser Doppler technique. Two recent large meta-analyses that have evaluated the effect of various forms of omega-3 PUFAs supplementation on human endothelial function brought conclusions that are not 
75 completely concordant. One meta-analysis reported that omega-3 PUFAs supplementation 76 significantly increased FMD of the brachial artery, and that this effect can be modified by the 77 health status of the participants, or by the dose of omega-3 PUFAs supplementation (Wang et al. 78 2012). On the contrary, a sensitivity analysis including only double-blinded, placebo-controlled 79 studies indicated that omega-3 PUFAs supplementation did not have a significant effect on 80 vascular endothelial function (Xin et al. 2012). Nevertheless, these analyses were limited by 81 significant heterogeneity in design of relevant studies, including the number of participants, 82 inclusion criteria such as age of participants or whether participants were healthy or diseased, 83 markers of endothelial function that were measured, dose and duration of omega-3 PUFAs 84 supplementation, forms of omega-3 PUFAs that were administered (ALA, EPA, DHA) alone or 85 in combination, and concomitant therapy that was used (Wang et al. 2012; Xin et al. 2012).

Functional food concept has been introduced only recently. This pertains to food of 87 natural origin that contains ingredients with a beneficial effect on human health (Drenjancevic et al. 2017). Food can be considered functional if it is shown to a satisfactory degree that, in addition to appropriate nutritional effects, it also has beneficial effects on one or more target functions of the body, in a way that it is important for improving the health condition and general

91 well-being or reducing risks of disease (Drenjancevic et al. 2017). It is important to emphasize

92 that functional food has to be actual food (not in the form of pills or capsules), and it has to show 93 its effects when consumed in normal daily amounts (Diplock et al. 1999). One of the most 94 common functional poultry products are hen eggs with increased content of desirable omega-3 PUFAs, which are available on the market. 
98

99

100

101

102

103

104

105

106

107

108

109

110

111

11230 years, normal body mass index (BMI), BP values and serum lipid ranges. Also, exclusion 113 criteria included a history of smoking, hypertension, coronary artery disease, diabetes,

114 hyperlipidemia, renal impairment, cerebrovascular and peripheral artery disease. None of the 115 subjects were taking drugs that could affect the endothelium and vascular function. Written 116 informed consent was obtained from each subject. The study protocol and procedures conformed 117 to the standards set by the latest revision of the Declaration of Helsinki and were approved by the 118 Ethical Committee of the Faculty of Medicine, University of Osijek (class: 602-04/14-08/06, 119 number: 2158-610714-114). 
Production of Omega-3 PUFAs Enriched Hen Eggs and Assessment of Fatty Acids Profile of

\section{Chicken Feed Mixtures and Edible Part of Eggs}

Omega-3 PUFAs enriched hen eggs were produced under a patent of Faculty of Agriculture, Josip Juraj Strossmayer University of Osijek. According to their protocol, common sunflower oil in feed mixtures fed to the laying hens was replaced with a mixture of fish $(1.33 \%)$, linseed $(1.33 \%)$, rapeseed $(1.33 \%)$ and soybean (1\%) oil. This replacement resulted in changed fatty acids profile in the laying hen's eggs that had reduced n-6 PUFAs and increased n3 PUFAs concentration, and thus a very favorable n-6/n-3 PUFAs ratio (2.63:1).

Assessment of fatty acids profile of chicken feed mixtures $(n=3)$ and edible part of eggs $(\mathrm{n}=10)$ was performed using gas liquid chromatography. Fat content of homogenized samples was extracted by using the method of Folch et al. (1957). All solvents used were ultrapure-grade by Sigma-Aldrich (Schnelldorf, Germany), and $100 \mathrm{mg} / \mathrm{L}$ butylated hydroxytoluene was added to the extraction mixture (chloroform/methanol $2 / 1 \mathrm{vol} / \mathrm{vol}$ ) as antioxidant. After this, fatty acid containing lipids were transmethylated by the base-catalyzed sodium-methoxide method of Christie (1982). Gas liquid chromatography was performed on a Shimadzu 2010 apparatus (Kyoto, Japan), equipped with a SP-2380 (Supelco, Bellefonte, USA) type capillary column (30 $\mathrm{m} \times 0.25 \mathrm{~mm}$ internal diameter, $0.20 \mu \mathrm{m}$ film) and flame ionization detector. To identify individual fatty acids in the chromatogram, a fatty acids standard mixture (Supelco 37 Component FAME Mix, CRM 47885) was used. Analyzed fatty acids are described in Table 2.

\section{Study Protocol}

This was a randomized, double-blind, placebo-controlled interventional study. None of the subjects had been taking omega-3 PUFAs enriched functional food nor omega-3 PUFAs 
142 supplementation in the form of capsules prior to enrollment in the present study. Subjects were 143 divided into two groups: control (Control) and experimental (OMEGA-3) group. The 144 experimental group consisted of 20 subjects (10 women and 10 men). Control group consisted of 14516 subjects ( 8 women and 8 men). Neither the researcher nor the subjects knew which group the 146 subjects belong to until the end of the three weeks' dietary protocol (eggs were labeled \#1 or \#2 147 before distributed to the Laboratory). During those three weeks the subjects ate three hen eggs 148 per day (total of 63 eggs). Subjects in OMEGA-3 group were given omega-3 PUFAs enriched 149 hen eggs (three per day; around $777 \mathrm{mg}$ of omega-3 PUFAs per day), while subjects in the 150 Control group received standard hen eggs produced on the same farm (three per day; around 277 151 mg of omega-3 PUFAs per day). Omega-3 PUFAs enriched eggs and standard hen eggs were the 152 same size (M commercial size). Subjects were instructed to boil the eggs for about 10 minutes 153 before consumption. Also, all subjects were instructed to take only the eggs given to them for the 154 purposes of the study (total of 63 eggs) and not to take the other food rich in omega-3 PUFAs, 155 omega-3 PUFAs enriched functional food or any other form of omega-3 PUFAs supplementation 156 during study protocol. The measurements were performed on the first and last day of the 157 protocol.

\section{Clinical Visits}

During the study, each subject had two clinical visits. The study protocol was performed

160 in the Laboratory for Clinical and Sports Physiology, Department of Physiology and 161 Immunology at Faculty of Medicine, University of Osijek. All testing occurred in the morning 162 after an overnight fasting. Subjects were instructed not to undertake any strenuous activity during 163 the $24 \mathrm{~h}$ preceding the visit and to avoid caffeine intake in the morning before the study visit. 

(MoorVMS-LDF, Axminster, UK) in response to vascular occlusion. LDF measurements were performed in a warm room (mean $\pm \mathrm{SD}$ temperature $\left.=23.5 \pm 0.5^{\circ} \mathrm{C}\right)$. Data collection started after 30 min of acclimatization to avoid temperature-related changes in blood flow. The subjects were resting in the supine position and the probe and wire were secured in place. The laser probe was attached to the subject's volar forearm skin $13-15 \mathrm{~cm}$ from the wrist at the place where basal blood flow was between 5 and 10 perfusion units (PU). LDF measurements were taken under

172 identical conditions, at the same time of the day, and the laser probe was attached to the 173 approximately same place at both study visits. changes in blood flow during post-occlusive reactive hyperemia (PORH) following release of an occlusion of blood flow (Crakowski et al. 2006). After the 5 min baseline measurement, vascular

177 occlusion was induced by inflating a pneumatic cuff on the upper arm to $30-50 \mathrm{mmHg}$ above the 178 systolic blood pressure (SBP). Measurements were taken before, during, and after release of 1 $179 \min (\mathrm{PORH}-1), 2 \mathrm{~min}$ (PORH-2) and $3 \mathrm{~min}$ occlusion (PORH-3), with 10 min interval between each occlusion to allow blood flow to return to baseline level. It is considered that such shorter 181 periods of vascular occlusion are at least partially mediated by endothelium-derived vasoactive 182 metabolites, rather than ischemia products (e.g. adenosine). Microcirculatory blood flow in a 183 given time was expressed in arbitrary PU and determined by software calculating the area under 184 the curve (AUC) during baseline flow, occlusion and reperfusion. Data were analyzed using appropriate software provided by the manufacturer of the LDF device (moorVMS-PC v4.0, 186 Axminster, UK). Because the flow does not reach the value of zero even when perfusion is 187 absent, flow values were expressed as a quotient of a standard comparator - baseline flow. The 
188 final result was expressed as the difference between percentage of flow change during 189 reperfusion and occlusion in relation to baseline (R-O \% increase). General procedures for LDF 190 PORH measurements were done according to the protocol already described in our laboratory 191 (Cavka et al. 2013; Cavka et al. 2015).

\section{Anthropometric Measurement}

Body mass index (BMI) was calculated as body mass (kg) divided by height (m) squared.

194 Waist and hip circumference were measured and waist-to-hip ratio (WHR) was calculated.

Arterial Blood Pressure Measurement

$\mathrm{BP}$ was measured at the beginning of each visit after a 15 min rest in the seated position

197 using an automatic oscillometric monitor (OMRON, Osaka, Japan). The final values of BP were 198 the mean of three repeated measurements. Mean arterial pressure (MAP) was calculated using a 199 formula of the SBP and the diastolic blood pressure (DBP): MAP $=[\mathrm{SBP}+2$ (DBP)]/3.

\section{Biochemical Laboratory Testing}

201

A venous blood sample was taken after 30 min resting in the supine position. Venous

202 blood samples were analyzed for fasting serum lipid profile (total cholesterol, high-density 203 lipoprotein (HDL) cholesterol, low-density lipoprotein (LDL) cholesterol, and triglycerides), 204 fasting blood glucose (fBG) and high-sensitivity C-reactive protein (hsCRP) using standard 205 laboratory methods at the Department of Clinical Laboratory Diagnostics, University Hospital 206 Osijek.

\section{Statistical Analysis}


All results are expressed as mean \pm standard deviation (SD). The sample size required to

209

210

211

212

213

214

215

216

217

218

219

220

221

222

223

224

225

226

227

228

229

show a potentially significant effect was calculated based on preliminary data in 10 subjects. To show an expected difference in LDF-measured microvascular blood flow change (R-O \%) before and after a specific diet protocol with alpha $=0.05$ and a statistical power of $80 \%$ for paired t-test, the needed sample size is 12 subjects per group. Clinical characteristics and all parameters measured before and after the specific study protocol were compared using a paired t-test. The normality of data distribution was assessed by the Kolmogorov-Smirnov normality test. The Wilcoxon rank-sum test was used when variables were not normally distributed. Student's t-test was used to compare parameters between the experimental groups. When variables were not normally distributed, the Mann-Whitney rank sum test was used. SigmaPlot, version11.2 (Systat Software, Inc., Chicago, IL, USA) was used for statistical analysis.

\section{Results}

\section{Content of Fatty Acids in Chicken Feed Mixture and Edible Part of Eggs}

Fatty acids profiles of chicken feed mixture (g/100 g of total fatty acids) and eggs (mg/100 g edible part) are described in Table 2. Each OMEGA-3 egg (average weight $58 \mathrm{~g}$ ) contained on average $259 \mathrm{mg}$ of omega-3 PUFAs (ALA $167 \mathrm{mg} / \mathrm{egg}$, EPA $7 \mathrm{mg} / \mathrm{egg}$, DHA 84 $\mathrm{mg} / \mathrm{egg}$ ). Each control egg (average weight $58 \mathrm{~g}$ ) produced on the same farm contained on average $92.4 \mathrm{mg}$ of omega-3 PUFAs.

\section{Characteristics of Study Population}

Characteristics of the study population are described in Table 1. There was no difference in participants' age between OMEGA-3 and Control group. Subjects of both sexes from 
230 OMEGA-3 and Control group were lean and had similar anthropometric measures (BMI and 231 WHR) before the study protocol (Table 1). No significant change in BMI and WHR in both 232 OMEGA-3 (BMI, kg/m² OMEGA-3 before 23.6 \pm 3.2 vs. after 23.6 $\pm 3.1, \mathrm{P}=0.979$; WHR 233 OMEGA-3 before $0.77 \pm 0.03$ vs. after $0.76 \pm 0.03, \mathrm{P}=0.500)$ and Control group of subjects (BMI, $234 \mathrm{~kg} / \mathrm{m}^{2}$ Control before $24.6 \pm 3.9$ vs. after $24.5 \pm 4.0, \mathrm{P}=0.576$; WHR Control before $0.77 \pm 0.03$ vs. 235 after $0.76 \pm 0.03, \mathrm{P}=0.303$ ) was induced by consumption of either three omega-3 PUFAs enriched 236 or three ordinary hen eggs over the course of three weeks.

Skin Microvascular Post-Occlusive Reactive Hyperemia (PORH) Response to Consumption of Omega-3 PUFAs Enriched Hen Eggs

PORH following all three vascular occlusion periods (PORH-1, PORH-2 and PORH-3) was significantly increased in OMEGA-3 group after the study protocol compared to before-diet measurement (Figure 1, Panel 1A). There was no change in skin microvascular PORH in the

242 Control group after the diet protocol compared to before-diet LDF measurement (Figure 1, Panel 243 1B).

BP measurements had demonstrated that all participants were normotensive before the

247 protocol. Table 3 presents BP changes before and after the study protocol in subjects from both 248 study groups. SBP, DBP and MAP were significantly decreased in OMEGA-3 group after the 249 protocol. In Control group, BP was not significantly changed before and after the study protocol. 
Table 4 presents changes in serum lipid profile, fBG and hsCRP values before and after

252 the corresponding study protocol in all subjects. There was no difference in any measured

253 biochemical parameters between the groups before the study protocol. Consumption of three

254 omega-3 PUFAs enriched hen eggs per day for three weeks did not induce a significant

255 difference in cholesterol, HDL cholesterol and LDL cholesterol level in OMEGA-3 group

256 compared to the pre-diet measurement. However, blood triglycerides levels were significantly

257 reduced after the study protocol in OMEGA-3 group compared to values measured before the

258 study. There was no difference in serum lipid profile values measured before and after the study

259 protocol in the Control group. fBG was not significantly changed before or after the specific

260 study protocol in both OMEGA-3 and Control group. hsCRP was significantly reduced in

261 OMEGA-3 group after the protocol compared to pre-diet measurement. There was no significant

262 change in hsCRP values after the study protocol in the Control group compared to values

263 measured before the protocol. However, the OMEGA-3 group, before and after treatment, had

264 lower hsCRP levels than the Control group.

\section{Discussion}

The salient findings of the present study are that consumption of three omega-3 PUFAs 268 enriched hen eggs per day (259 mg omega-3 PUFAs/egg: ALA 167 mg/egg, EPA 7 mg/egg, 269 DHA $84 \mathrm{mg} / \mathrm{egg} ; 777 \mathrm{mg}$ omega-3 PUFAs/day) for three weeks: 1) significantly enhanced skin 270 microvascular reactivity to vascular occlusion; 2) significantly decreased arterial BP; 3)

271 significantly decreased blood triglycerides level; and 4) significantly decreased hsCRP level 272 compared to baseline in young healthy population. These findings are in contrast to those 273 pertaining to standard eggs consumed by the control group, which showed no change in 
274 measured parameters after 3 weeks. It should be emphasized that the present study (randomized,

275 double-blind, placebo-controlled interventional study) is one of the few studies to investigate the

276 effect of omega-3 PUFAs supplementation in the form of functional food (omega-3 PUFAs

277 enriched hen eggs) on traditional CV risk factors, and the first one that also investigated its effect

278 on microvascular function in young healthy subjects.

\section{The Effect of Omega-3 PUFAs on Vascular Function}

Omega-3 PUFAs alter vascular and endothelial function by incorporating into its membrane phospholipids in which signaling molecules and receptors for endothelial cell

282 function are located (Endo and Arita, 2016). Omega-3 PUFAs may increase NO bioavailability

283 directly by stimulating endothelial nitric oxide synthase (eNOS) gene and protein expression

284 (Gortan Cappellari et al. 2013; Lopez et al. 2004; Zhang et al. 2013), or indirectly by attenuating

285 reactive oxygen species (ROS) and thus decreasing oxidative stress level, as demonstrated in cell

286 cultures and isolated blood vessels (Gortan Cappellari et al. 2013; Zhang et al. 2013). Omega-3

287 PUFAs may also attenuate cellular and systemic inflammation by reducing soluble cell adhesion

288 molecules (sCAMs), IL-6 and/or CRP level (Wang et al. 2011).

Functional vascular studies, focused on peripheral macrovasculature (e.g. brachial artery FMD), strongly suggested that omega-3 PUFAs supplementation (EPA+DHA, DHA alone or ALA alone) improves vascular and endothelial function in CV disease patients and in patients at high CV risk (Yagi et al. 2015; Tousoulis et al. 2014; Egert et al. 2014; Merino et al. 2014). On

294 EPA+DHA capsules) on vascular endothelial function in healthy individuals provided 295 inconsistent results (Siasos et al. 2013; Rizza et al. 2009; Shah et al. 2007). Some authors 296 reported that omega-3 PUFAs supplementation improved brachial artery FMD in healthy 
297 subjects, while others failed to demonstrate such effect (Sanders et al. 2011; Singhal et al. 2013;

298 Skulas-Ray et al. 2011). One study demonstrated that omega-3 PUFAs supplementation 299 (EPA+DHA capsules for 12 months) amounting to less than $1.8 \mathrm{~g} /$ day $(0.45$ and $0.9 \mathrm{~g} /$ day $)$ does 300 not significantly improve FMD in healthy adults (Sanders et al. 2011). Supplementation of 301 omega-3 PUFAs in the form of EPA+DHA capsules (1 $\mathrm{g}$ in only one dose or $4 \mathrm{~g} /$ day during 302 period of 4 weeks) significantly decreased postprandial endothelial dysfunction (Miyoshi et al. 303 2014; Fahs et al. 2010). On the contrary, a single Mediterranean meal (rich in omega-3 PUFAs) 304 did not alter FMD in healthy men (Lacroix et al. 2016). Two studies reported that omega-3 305 PUFAs supplementation (Mediterranean diet for 4 weeks or a single fish oil meal) improved 306 postprandial endothelial microvascular reactivity assessed by LDF (Fuentes et al. 2008; Armah 307 et al. 2008). Only one study demonstrated that fish oil supplementation for 8 months increased 308 microvascular reactivity in normal healthy subjects (Khan et al. 2003). In the present study, 309 healthy individuals who consumed three omega-3 PUFAs enriched eggs (ALA+EPA+DHA) for 310 three weeks exhibited improved PORH in skin microcirculation, in all three vascular occlusion 311 periods (Figure 1).

\section{The Effect of Omega-3 PUFAs on Blood Pressure}

314 in lowering arterial BP in hypertensive patients (Minihane et al. 2016). The present study is one 315 of very few studies reporting that consumption of omega-3 PUFAs enriched hen eggs for three 316 weeks decreased arterial BP in generally normotensive and healthy young population (Table 3).

317 These results are in concordance with the study by Oh et al., who reported that consumption of 4 318 omega-3 enriched eggs for 4 weeks decreased BP level in healthy individuals (Oh et al. 1991). 319 Such decrease in BP level was not seen when omega-3 PUFAs were supplemented in the form of 
320 four fish servings per week ( 800 mg/serving EPA+DHA for 8 weeks $)$ or fish oil 321 supplementation (2 g/day EPA+DHA for 12 weeks; $1.7 \mathrm{~g} /$ day EPA+DHA for 4 weeks) to 322 normotensive individuals (Grieger et al. 2014; Hlais et al. 2013; Root et al. 2013). Omega-3 323 PUFAs supplementation (EPA+DHA capsules $4 \mathrm{~g}$ /day) for 12 weeks diminished increases in 324 mean arterial BP and DBP at the onset of handgrip exercise and therefore reduced CV response 325 to acute physiological stress in young and older healthy subjects, although no change in resting 326 BP after omega-3 PUFAs supplementation was detected (Clark et al. 2016). Furthermore, a 327 beneficial effect of two weeks $3 \mathrm{~g}$ /day omega-3 PUFAs supplementation (fish oil or oily fish) on 328 hemodynamic parameters was reported in patients with uncontrolled hypertension and patients 329 older than 45 years, but the same effect was also noticed in normotensive subjects (Cabo et al. 330 2012). It was suggested that such inconsistency in observed results may reflect genetic variation 331 associated with BP (AlSaleh et al. 2014; Ellulu et al. 2016).

\section{Anti-inflammatory Effect of Omega-3 PUFAs}

The present study has shown a significant decrease of hsCRP compared to baseline in the study group consuming omega-3 PUFAs eggs (ALA+EPA+DHA), while such effect was not observed in subjects consuming standard hen eggs (Table 4). This is in agreement with studies showing that omega-3 PUFAs supplementation (fish oil capsules with $300 \mathrm{mg}$ EPA and $200 \mathrm{mg}$ DHA per day for 8 weeks) decreased hsCRP in hypertensive, obese and diabetic patients (Ellulu et al. 2016), as well as in heathy adults (0.85 g EPA + 3.4 g DHA per day for 8 weeks) (SkulasRay et al. 2011). On the other hand, some authors did not detect any significant effect of a moderate dose of omega-3 PUFAs (fish oil supplementation $1400 \mathrm{mg}$ EPA+DHA per day for 18 weeks) on CRP and IL-6 in healthy adults (Muldoon et al. 2016), nor was any positive effect of 
342 fish oil omega-3 PUFAs (1.7 g EPA+DHA packets per day for 4 weeks) on arterial health and

343 inflammatory markers observed in young but overweight population (Root et al. 2013).

\section{The Effect of Omega-3 PUFAs on Lipid Profile}

It is known that omega-3 PUFAs reduce serum triglyceride concentrations by inhibiting two crucial enzymes involved in hepatic triglycerides biosynthesis, and by increasing very lowdensity lipoprotein (VLDL) clearance in the peripheral circulation (Jacobson 2008). A large body of evidence reported the clinical relevance of omega-3 PUFAs in the management of all forms of persistent hypertriglyceridemia and high triglycerides in patients with increased CV risk (Zulyniak et al. 2016). The authors of the present study found two studies that investigated the effect of omega-3 PUFAs enriched eggs on serum lipids in healthy individuals. Similarly as in the present study, one study demonstrated that consumption of 4 omega-3 PUFAs enriched eggs per day for 4 weeks significantly decreased mean plasma triglyceride concentration in healthy subjects, while consumption of control eggs had the opposite effect (Oh et al. 1991). Furthermore, another study reported that omega-3 PUFAs enriched eggs $(\sim 500 \mathrm{mg}$ DHA +40 mg EPA + 1 g ALA per egg; 6 eggs/week for 8 weeks) and a walnut-supplemented diet (2.95 g ALA/28.4 g walnuts, 6 times/week) had a beneficial effect on serum lipids compared to standard eggs in healthy lacto-ovo-vegetarians, which is in concordance with the results of the present study (Burns-Whitmore et al. 2014). The results of the present study are also in agreement with others demonstrating the triglyceride-lowering benefits associated with fish oil supplements (EPA+DHA) in children, young and older men (960 mg/day for 16 weeks), as well as in healthy older women (3 g/day for 12 weeks) (Logan \& Spriet 2015; Pase et al. 2015; Damsgaard et al. 2013). In contrast, several studies failed to demonstrate a relationship between fish-oil supplementation (2 g/day EPA+DHA for 12 weeks; $1.7 \mathrm{~g} /$ day EPA+DHA for 4 weeks) and lipid 
365 profile in healthy individuals (Hlais et al. 2013; Root et al. 2013). This discrepancy could be 366 explained by heterogeneity in design of these studies in terms of participants' age as well as 367 dose, duration and forms of omega-3 PUFAs supplementation.

The strengths of the present study are the following: a) the fact that it is designed as a randomized double-blind placebo-controlled study, b) homogeneity in health status between the 371 intervention and control groups, and c) usage of a natural, cheap and accessible dietary source of omega-3 PUFAs supplementation.

One limitation of this study is the authors' technical inability to measure blood fatty acid 374 profile, since that method is not available at the institutions where the authors work. However, 375 considering the stark and significant differences demonstrated by the results of the present study, 376 as well as the strict instructions that had been given to the subjects in regard of diets, the authors 377 of the present study are confident that the obtained differences are the consequence of the dietary 378 omega-3 PUFAs intake during the study protocol. Another possible limitation of the present 379 study is that consumption of three eggs per day is quite excessive. Since 'functional food should 380 show effects when consumed in normal daily amounts', future studies based on a populations' 381 habitual weekly omega-3 PUFAs rich food intake should be conducted to confirm the findings. 382 Also, it is important to conduct further investigation of the underlying mechanisms involved in 383 CV health background, and to include not only healthy individuals but also CV patients, which 384 would put the results in the context of reduced risk of developing pathology. 
386 In conclusion, the present study provides evidence that omega-3 PUFAs from functional food

387 (omega-3 PUFAs enriched eggs) increased microvascular reactivity in young healthy subjects,

388 and that they increased serum lipid values by decreasing blood triglyceride levels in young

389 healthy individuals. The study has also demonstrated that consumption of omega-3 PUFAs

390 enriched eggs has the potential to decrease BP level and hsCRP in young healthy subjects. The

391 present study is the first one to investigate the effect that the incorporating of n-3 PUFAs

392 enriched eggs in everyday eating habits has on microvascular reactivity. The present study has

393 significant clinical and public health relevance, and it is also important for further investigation

394 of the underlying mechanisms involved in CV health background.

396 Acknowledgments

397 This study is supported by the European Structural and Investment Funds grant for the Croatian

398 National Scientific Center of Excellence for Personalized Health Care, University of Josip Juraj

399 Strossmayer Osijek (grant \#KK.01.1.1.01.0010).

401 Conflicts of Interests

402 The authors declare no conflict of interest. 
405

406

407

408

409

410

411

412

413

414

415

416

417

418

419

420

421

422

423

424

425

426
AlSaleh, A., Maniou, Z., Lewis, F.J., Hall, W.L., Sanders, T.A., O'Dell, S.D. et al. 2014. Interaction between a CSK gene variant and fish oil intake influences blood pressure in healthy adults. J. Nutr. 144(3): 267-72. doi: 10.3945/jn.113.185108.

Armah, C. K., Jackson, K. G., Doman, I., James, L., Cheghani, F., Minihane, A.M. 2008.

Fish oil fatty acids improve postprandial vascular reactivity in healthy men. Clin. Sci.

(Lond). 114(11): 679-86.

Auger, C., Said, A., Nguyen, P.N. Chabert, P., Idris-Khodja, N., Schini-Kerth, V.B. 2016.

Potential of food and natural products to promote endothelial and vascular health. J

Cardiovasc. Pharmacol. 68(1): 11-8. doi: 10.1097/FJC.0000000000000382.

Burns-Whitmore, B., Haddad, E., Sabaté, J., Rajaram, S. 2014. Effects of supplementing n-3 fatty acid enriched eggs and walnuts on cardiovascular disease risk markers in healthy freeliving lacto-ovo-vegetarians: a randomized, crossover, free-living intervention study. Nutr. J. 13: 29. doi: 10.1186/1475-2891-13-29.

Cabo, J., Alonso, R. \& Mata, P. 2012. Omega-3 fatty acids and blood pressure. Br. J. Nutr. 2: S195-200. doi: 10.1017/S0007114512001584.

Cavka, A., Cosic, A., Grizelj, I., Koller, A., Jelaković, B., Lombard, J.H., et al. 2013. Effects of AT1 receptor blockade on plasma thromboxane A2 (TXA2) level and skin microcirculation in young healthy women on low salt diet. Kidney. Blood. Press. Res. 37(45): 432-42. doi: 10.1159/000355723.

Cavka, A., Cosic, A., Jukic, I., Jelakovic, B., Lombard, J.H., Phillips, S.A., et al. 2015. The role of cyclo-oxygenase-1 in high-salt diet-induced microvascular dysfunction in humans. J. Physiol. 593(24): 5313-24. doi: 10.1113/JP271631. 
427

428

429

430

431

432

433

434

435

436

437

438

439

440

441

442

443

444

445

446

447

448

Christie, W.W. 1982. A simple procedure for rapid transmethylation of glycerolipids and cholesteryl esters. J. Lipid. Res. 23: 1072-1075.

Clark, C. M., Monahan, K. D. \& Drew, R. C. 2016. Omega-3 polyunsaturated fatty acid supplementation attenuates blood pressure increase at onset of isometric handgrip exercise in healthy young and older humans. Physiol. Rep. 4(14): e12875. doi: 10.14814/phy2.12875.

Crakowski, J. L., Minson, C. T., Salvat-Melis, M., Halliwill JR. 2006. Methodological issues in the assessment of skin microvascular endothelial function in humans. Trends. Pharmacol. Sci. 27(9): 503-8. doi: 10.1016/j.tips.2006.07.008.

Damsgaard, C. T., Stark, K. D., Hjorth, M. F., Biltoft-Jensen, A., Astrup, A., Michaelsen, K.F., et al. 2013. n-3 PUFA status in school children is associated with beneficial lipid profile, reduced physical activity and increased blood pressure in boys. Br. J. Nutr. 110(7): 1304-12. doi: 10.1017/S0007114513000585.

Diplock, A., Aggett, P., Ashwell, M., Bornet, F., Fern, E., Roberfroid, M. 1999. Scientific concepts of functional foods in Europe. Consensus document. Br. J. Nutr. 81(Suppl 1): S127.

Drenjancevic, I., Kralik, G., Kralik, Z., Mihalj, M., Stupin, A., Novak, S., et al. 2017. The Effect of Dietary Intake of Omega-3 Polyunsaturated Fatty Acids on Cardiovascular Health: Revealing Potentials of Functional Food. In Superfood and Functional Food - The Development of Superfoods and Their Roles as Medicine. Edited by Shiomi N and Waisundara V. IN TECH d.o.o, Rijeka, Croatia. pp. 207-232.

Egert, S., Baxheinrich, A., Lee-Barkey, Y.H., Tschoepe, D., Wahrburg, U., Stratmann, B. 2014. Effects of an energy-restricted diet rich in plant-derived $\alpha$-linolenic acid on systemic 
449 inflammation and endothelial function in overweight-to-obese patients with metabolic $450 \quad$ syndrome traits. Br. J. Nutr. 112(8): 1315-22. doi: 10.1017/S0007114514002001.

451 Ellulu, M.S., Khaza'ai, H., Patimah, I., Rahmat, A., Abed, Y. 2016. Effect of long chain 452 omega-3 polyunsaturated fatty acids on inflammation and metabolic markers in hypertensive 453 and/or diabetic obese adults: a randomized controlled trial. Food. Nutr. Res. 60: 29268. doi: $454 \quad 10.3402 /$ fnr.v60.29268.

455 Endo, J. \& Arita, M. 2016. Cardioprotective mechanism of omega-3 polyunsaturated fatty 456 acids. J. Cardiol. 67(1): 22-7. doi: 10.1016/j.jjcc.2015.08.002.

457 Fahs, C.A., Yan, H., Ranadive, S., Rossow, L.M., Agiovlasitis, S., Wilund, K.R., et al. 2010. 458 The effect of acute fish-oil supplementation on endothelial function and arterial stiffness 459 following a high-fat meal. Appl. Physiol. Nutr. Metab. 35(3): 294-302. doi: 10.1139/H10$460 \quad 020$. Folch, J. M., Lees, M. \& Sloane-Stanley, G. H. 1957. A simple method for the isolation and 462 purification of total lipids from animal tissues. J. Biol. Chem. 226: 495-509.

463 Fraeye, I., Bruneela, C., Lemahieua, C., Buyse, J., Muylaert, K., Foubertet, I. 2012. Dietary 464 enrichment of eggs with omega-3 fatty acids: a review. Food. Res. Int. 48: 961-969. 465 doi:10.1016/j.foodres.2012.03.014.

466 Fuentes, F., López-Miranda, J., Pérez-Martínez, P., Jiménez, Y., Marín, C., Gómez, P., et al. 467 2008. Chronic effects of a high-fat diet enriched with virgin olive oil and a low-fat diet 468 enriched with alpha-linolenic acid on postprandial endothelial function in healthy men. Br. J. $469 \quad$ Nutr. 100(1): 159-65. doi: 10.1017/S0007114508888708. 
Gortan Cappellari, G., Losurdo, P., Mazzucco, S., Panizon, E., Jevnicar, M., Macaluso, L., et

471

472

473

474

475

476

477

478

479

480

481

482

483

484

485

486

487

488

489

490

491

al. 2013. Treatment with n-3 polyunsaturated fatty acids reverses endothelial dysfunction and oxidative stress in experimental menopause. J. Nutr. Biochem. 24(1): 371-9. doi: 10.1016/j.jnutbio.2012.07.012.

Grieger, J. A., Miller, M. D. \& Cobiac, L. 2014. Investigation of the effects of a high fish diet on inflammatory cytokines, blood pressure, and lipids in healthy older Australians. Food. Nutr. Res. 58. doi: 10.3402/fnr.v58.20369.

Hlais, S., El-Bistami, D., El Rahi, B., Mattar, M.A., Obeid, O.A. 2013. Combined fish oil and high oleic sunflower oil supplements neutralize their individual effects on the lipid profile of healthy men. Lipids, 48(9): 853-61. doi: 10.1007/s11745-013-3819-x.

Jacobson, T. A. 2008. Role of n-3 fatty acids in the treatment of hypertriglyceridemia and cardiovascular disease. Am. J. Clin. Nutr. 87(6): 1981S-90S. doi: 10.1093/ajcn/87.6.1981S

Khan, F., Elherik, K., Bolton-Smith, C., Barr, R., Hill, A., Murrie, I., et al. 2003. The effects of dietary fatty acid supplementation on endothelial function and vascular tone in healthy subjects. Cardiovasc. Res. 59(4): 955-62.

Lacroix, S., Des Rosiers, C., Gayda, M., Nozza, A., Thorin, É., Tardif, J.C., et al. 2016. A single Mediterranean meal does not impair postprandial flow-mediated dilatation in healthy men with subclinical metabolic dysregulations. Appl. Physiol. Nutr. Metab. 41(8): 888-94. doi: 10.1139/apnm-2015-0490.

Lewis, N. M., Schalch, K., Scheideler, S. E. 2000. Serum lipid response to n-3 fatty acid enriched eggs in persons with hypercholesterolemia. J. Am. Dietetic. Assoc. 100: 365-367. doi: 10.1016/S0002-8223(00)00111-5 
492

493

494

495

496

497

498

499

500

501

502

503

504

505

506

507

508

509

510

511

512

513

Logan, S. L. \& Spriet, L. L. 2015. Omega-3 Fatty Acid Supplementation for 12 Weeks Increases Resting and Exercise Metabolic Rate in Healthy Community-Dwelling Older Females. PLoS One, 10(12): e0144828. doi: 10.1371/journal.pone.0144828.

Lopez, D., Orta, X., Casos, K., Sáiz, M.P., Puig-Parellada, P., Farriol, M., et al. 2004. Upregulation of endothelial nitric oxide synthase in rat aorta after ingestion of fish oil-rich diet. Am. J. Physiol. Heart. Circ. Physiol. 287(2): H567-72. doi:

10.1152/ajpheart.01145.2003.

Maki, K. C., Van Elswyk, M. E., McCarthy, D., Seeley, M.A., Veith, P.E., Hess, S.P., et al. 2003. Lipid responses in mildly hypertriglyceridemic men and women to consumption of docosahexaenoic acid-enriched eggs. Int. J. Vitam. Nutr. Res. 73: 357-368. doi: $10.1024 / 0300-9831.73 .5 .357$

Merino, J., Sala-Vila, A., Kones, R., Ferre, R., Plana, N., Girona, J., et al. 2014. Increasing long-chain n-3 PUFA consumption improves small peripheral artery function in patients at intermediate-high cardiovascular risk. J. Nutr. Biochem. 25(6): 642-6. doi: 10.1016/j.jnutbio.2014.02.004.

Minihane, A. M., Armah, C. K., Miles, E. A., Madden, J.M., Clark, A.B., Caslake, M.J., et al. 2016. Consumption of Fish Oil Providing Amounts of Eicosapentaenoic Acid and Docosahexaenoic Acid That Can Be Obtained from the Diet Reduces Blood Pressure in Adults with Systolic Hypertension: A Retrospective Analysis. J. Nutr. 146(3): 516-23. doi: $10.3945 /$ jn.115.220475.

Miyoshi, T., Noda, Y., Ohno, Y., Sugiyama, H., Oe, H., Nakamura, K., et al. 2014. Omega-3 fatty acids improve postprandial lipemia and associated endothelial dysfunction in healthy 
514 individuals - a randomized cross-over trial. Biomed. Pharmacother. 68(8): 1071-7. doi:

$515 \quad$ 10.1016/j.biopha.2014.10.008.

516 Muldoon, M. F., Laderian, B., Kuan, D.C., Sereika, S.M., Marsland, A.L., Manuck, S.B.

517 2016. Fish oil supplementation does not lower C-reactive protein or interleukin-6 levels in

518 healthy adults. J. Intern. Med. 279(1): 98-109. doi: 10.1111/joim.12442.

519 Oh, S.Y., Ryue, J., Hsieh, C.H., Bell, D.E. 1991. Eggs enriched in omega-3 fatty acids and 520 alterations in lipid concentrations in plasma and lipoproteins and in blood pressure. Am. J.

521 Clin. Nutr. 54(4): 689-95. doi: 10.1093/ajcn/54.4.689

522 Pase, M. P., Grima, N., Cockerell, R., Stough, C., Scholey, A., Sali, A., et al. 2015. The 523 effects of long-chain omega-3 fish oils and multivitamins on cognitive and cardiovascular 524 function: a randomized, controlled clinical trial. J. Am. Coll. Nutr. 34(1): 21-31. doi:

$525 \quad 10.1080 / 07315724.2014 .880660$.

526 Rizza, S., Tesauro, M., Cardillo, C., Galli, A., Iantorno, M., Gigli, F., et al. 2009. Fish oil 527 supplementation improves endothelial function in normoglycemic offspring of patients with 528 type 2 diabetes. Atherosclerosis, 206(2): 569-74. doi: 10.1016/j.atherosclerosis.2009.03.006.

529 Root, M., Collier, S.R., Zwetsloot, K. A., West, K.L., McGinn, M.C. 2013. A randomized 530 trial of fish oil omega-3 fatty acids on arterial health, inflammation, and metabolic syndrome 531 in a young healthy population. Nutr. J., 12: 40. doi: 10.1186/1475-2891-12-40.

532 Ross, R. 1999. Atherosclerosis - an inflammatory disease. N. Engl. J. Med. 340: 115-126. 
Sanders, T. A., Hall, W. L., Maniou, Z., Lewis, F., Seed, P.T., Chowienczyk, P.J. 2011.

Effect of low doses of long-chain n-3 PUFAs on endothelial function and arterial stiffness: a randomized controlled trial. Am. J. Clin. Nutr. 94(4): 973-80. doi: 10.3945/ajen.111.018036. Cardiovascular and endothelial effects of fish oil supplementation in healthy volunteers. J. Cardiovasc. Pharmacol. Ther. 12(3): 213-9. doi: 10.1177/1074248407304749.

Siasos, G., Tousoulis, D., Oikonomou, E., Zaromitidou, M., Verveniotis, A., Plastiras, A., et al. 2013. Effects of $\Omega-3$ fatty acids on endothelial function, arterial wall properties, inflammatory and fibrinolytic status in smokers: a cross over study. Int. J. Cardiol. 166(2): 340-6. doi: 10.1016/j.ijcard.2011.10.081.19.

Singhal, A., Lanigan, J., Storry, C., Low, S., Birbara, T., Lucas, A., et al. 2013.

Docosahexaenoic acid supplementation, vascular function and risk factors for cardiovascular disease: a randomised controlled trial in young adults. J. Am. Heart. Assoc. 2(4): e000283. doi: 10.1161/JAHA.113.000283.

Skulas-Ray, A. C., Kris-Etherton, P. M., Harris, W. S., Vanden Heuvel, J.P., Wagner, P.R., West, S.G. 2011. Dose-response effects of omega-3 fatty acids on triglycerides, inflammation, and endothelial function in healthy persons with moderate hypertriglyceridemia. Am. J. Clin. Nutr. 93(2): 243-52. doi: 10.3945/ajcn.110.003871.

Tousoulis, D., Plastiras, A., Siasos, G., Oikonomou, E., Verveniotis, A., Kokkou, E., et al. 2014. Omega-3 PUFAs improved endothelial function and arterial stiffness with a parallel antiinflammatory effect in adults with metabolic syndrome. Atherosclerosis, 232(1): 10-6. doi: 10.1016/j.atherosclerosis.2013.10.014. 
Wang, Q., Liang, X., Wang, L., Lu, X., Huang, J., Cao, J., et al. 2012. Effect of omega-3 fatty acids supplementation on endothelial function: a meta-analysis of randomized controlled trials. Atherosclerosis, 221(2): 536-43. doi: 10.1016/j.atherosclerosis.2012.01.006.

Wang, T. M., Chen, C. J., Lee, T. S., Chao, H.Y., Wu, W.H., Hsieh, S.C., et al. 2011. Docosahexaenoic acid attenuates VCAM-1 expression and NF-kappaB activation in TNFalpha-treated human aortic endothelial cells. J. Nutr. Biochem. 22(2): 187-94. doi: 10.1016/j.jnutbio.2010.01.007.

Xin, W., Wei, W. \& Li, X. 2012. Effect of fish oil supplementation on fasting vascular endothelial function in humans: a meta-analysis of randomized controlled trials. PloS one, 7(9): e46028. doi: 10.1371/journal.pone.0046028.

Yagi, S., Aihara, K., Fukuda, D., Takashima, A., Hara, T., Hotchi, J., et al. 2015. Effects of docosahexaenoic Acid on the endothelial function in patients with coronary artery disease. J. Atheroscler. Thromb. 22(5): 447-54. doi: 10.5551/jat.26914.

Zhang, W., Fu, F., Tie, R., Liang, X., Tian, F., Xing, W., et al. 2013. Alpha-linolenic acid intake prevents endothelial dysfunction in high-fat diet-fed streptozotocin rats and underlying mechanisms. Vasa, 42(6): 421-8. doi: 10.1024/0301-1526/a000311.

Zulyniak, M. A., Roke, K., Gerling, C., Logan, S.L., Spriet, L.L., Mutch, D.M. 2016. Fish oil regulates blood fatty acid composition and oxylipin levels in healthy humans: A comparison of young and older men. Mol. Nutr. Food. Res. 60(3): 631-41. doi: 10.1002/mnfr.201500830. 


\section{Tables}

Table 1. Characteristics of study population at baseline

\begin{tabular}{|c|c|c|c|c|}
\hline $\begin{array}{l}\text { Group } \\
\text { Parameter }\end{array}$ & \multicolumn{2}{|c|}{ OMEGA-3 } & \multicolumn{2}{|c|}{ Control } \\
\hline $\mathbf{n}$ & \multicolumn{2}{|c|}{20} & \multicolumn{2}{|c|}{16} \\
\hline $\operatorname{sex}, M / W$ & \multicolumn{2}{|c|}{$10 / 10$} & \multicolumn{2}{|c|}{$8 / 8$} \\
\hline \multirow[t]{2}{*}{ age, years } & \multicolumn{2}{|c|}{$21 \pm 1$} & \multicolumn{2}{|c|}{$21 \pm 1$} \\
\hline & M & W & M & W \\
\hline BMI, kg/m² & $24.7 \pm 2.3$ & $20.8 \pm 1.7$ & $24.3 \pm 2.1$ & $20.9 \pm 1.8$ \\
\hline WHR & $0.79 \pm 0.03$ & $0.77 \pm 0.05$ & $0.81 \pm 0.01$ & $0.76 \pm 0.04$ \\
\hline
\end{tabular}

Results are presented as mean $\pm \mathrm{SD}$.

n- number of participants; M - men; W - women; BMI - body mass index; WHR - waist-to-hip ratio

579

580

581

582

583

584

585

586

587

588

589

590

591

592

593 
Table 2. Fatty acids profile of hens' feeding mixtures and edible part of eggs

\begin{tabular}{|c|c|c|c|c|c|}
\hline \multirow{3}{*}{ Fatty acid } & & \multicolumn{2}{|c|}{ Feeding Mixture $(n=3)$} & \multicolumn{2}{|c|}{$\operatorname{Eggs}(n=10)$} \\
\hline & & Control & OMEGA-3 & Control & OMEGA-3 \\
\hline & & \multicolumn{2}{|c|}{$\mathrm{g} / 100 \mathrm{~g}$ of total fatty acids } & \multicolumn{2}{|c|}{$\mathrm{mg} / 100 \mathrm{~g} \mathrm{egg}^{1}$} \\
\hline$\sum$ SFA & & $20.32 \pm 0.20$ & $16.38 \pm 0.07 *$ & $2432.21 \pm 79.33$ & $2184.33 \pm 44.30 *$ \\
\hline$\sum$ MUFA & & $28.47 \pm 0.08^{*}$ & $34.20 \pm 0.05$ & $2929.57 \pm 143.06$ & $2911.51 \pm 75.23$ \\
\hline$\sum$ n-6 PUFA & & $46.72 \pm 0.15$ & $38.45 \pm 0.06^{*}$ & $1442.37 \pm 133.95$ & $1343.50 \pm 57.56$ \\
\hline \multirow{6}{*}{$\sum$ n-3 PUFA } & LA & $39.22 \pm 1.20$ & $35.65 \pm 0.62 *$ & $1340.32 \pm 131.25$ & $1243.27 \pm 57.73$ \\
\hline & AA & $0 \pm 0.00^{*}$ & $0.10 \pm 0.02$ & $39.84 \pm 4.88$ & $42.59 \pm 3.25$ \\
\hline & & $4.50 \pm 0.07 *$ & $10.97 \pm 0.07$ & $182.14 \pm 12.65 *$ & $510.35 \pm 16.25$ \\
\hline & ALA & $4.09 \pm 0.06^{*}$ & $8.33 \pm 0.05$ & $81.96 \pm 10.19 *$ & $329.78 \pm 14.30$ \\
\hline & EPA & $0 \pm 0.00 *$ & $0.88 \pm 0.03$ & $4.20 \pm 1.07^{*}$ & $13.94 \pm 2.17$ \\
\hline & DHA & $0.40 \pm 0.07 *$ & $1.59 \pm 0.06$ & $94.57 \pm 9.13 *$ & $165.24 \pm 12.63$ \\
\hline$\sum n-6 / \sum n-31$ & UFA & & & & \\
\hline
\end{tabular}

Results are presented as mean $\pm \mathrm{SD}$.

n- number of analysis; SD- standard deviation;

¿SFA- saturated fatty acids (C14:0, C15:0, C16:0, C17:0, C18:0, C20:0, C21:0, C23:0);

¿MUFA- monounsaturated fatty acids (C14:1, C16:1, C18:1n9t, C18:1n9c, C20:1n9, C22:1n9);

$\sum \mathrm{n}-6$ PUFA- polyunsaturated fatty acids (C18:2n6c, C18:3n6, C20:3n6, C20:4n6, C22:2n6); LA- linoleic acid

(C18:2n6c); AA- arachidonic acid (C20:4n6);

Sn-3 PUFA- polyunsaturated fatty acids (C18:3n3, C20:3n3, C20:5n3, C22:6n3);

ALA- alpha linolenic acid (C18:3n3); EPA- eicosapentaenoic acid (C20:5n3); DHA- docosahexaenoic acid (C22:6n3);

594

1 edible part; $* \mathrm{P}<0.05$ Control vs. OMEGA-3

595

596

597

598

599

600

601

602

603

604 
Table 3. Arterial blood pressure of study population

\begin{tabular}{|c|c|c|c|c|}
\hline \multirow[t]{2}{*}{ group } & \multicolumn{2}{|c|}{ OMEGA-3 } & \multicolumn{2}{|c|}{ Control } \\
\hline & before & after 3 weeks & before & after 3 weeks \\
\hline $\mathbf{n}$ & \multicolumn{2}{|c|}{20} & \multicolumn{2}{|c|}{16} \\
\hline SBP, mmHg & $118 \pm 8$ & $111 \pm 8 *$ & $120 \pm 10$ & $122 \pm 8$ \\
\hline DBP, $\mathrm{mmHg}$ & $70 \pm 8$ & $67 \pm 7 *$ & $73 \pm 7$ & $75 \pm 5$ \\
\hline MAP, $\mathrm{mmHg}$ & $86 \pm 7$ & $83 \pm 7 *$ & $89 \pm 8$ & $91 \pm 5$ \\
\hline
\end{tabular}

Results are presented as mean \pm SD.

$\mathrm{n}$ - number of participants; SBP - systolic blood pressure; DBP - diastolic blood pressure; MAP - mean arterial pressure

* $\mathrm{P}<0.05$ OMEGA-3 before vs. after 3 weeks

605

606

607

608

609

610

611

612

613

614

615

616

617

618

619

620

621

622 
Table 4. Fasting biochemical parameters of study population

\begin{tabular}{lcccc}
\hline \multirow{2}{*}{ group } & \multicolumn{2}{c}{ OMEGA-3 } & \multicolumn{2}{c}{ Control } \\
& before & after 3 weeks & before & after 3 weeks \\
\hline $\mathbf{n}$ & \multicolumn{3}{c}{20} & \multicolumn{2}{c}{16} \\
cholesterol, mmol/L & $4.6 \pm 0.8$ & $4.6 \pm 0.7$ & $4.2 \pm 0.6$ & $4.2 \pm 0.7$ \\
triglycerides, mmol/L & $1.2 \pm 0.5$ & $1.0 \pm 0.5^{*}$ & $1.0 \pm 0.5$ & $0.9 \pm 0.3$ \\
HDL cholesterol, mmol/L & $1.6 \pm 0.4$ & $1.6 \pm 0.4$ & $1.3 \pm 0.2$ & $1.3 \pm 0.3$ \\
LDL cholesterol, mmol/L & $2.7 \pm 0.6$ & $2.7 \pm 0.5$ & $2.6 \pm 0.5$ & $2.5 \pm 0.5$ \\
& & & & \\
fBG, mmol/L & $5.4 \pm 0.9$ & $5.1 \pm 0.7$ & $5.0 \pm 0.6$ & $5.2 \pm 0.5$ \\
hsCRP, mg/L & $1.1 \pm 1.4$ & $0.8 \pm 0.9^{*}$ & $1.8 \pm 2.6$ & $1.9 \pm 2.9$ \\
\hline
\end{tabular}

Results are presented as mean \pm SD.

$\mathrm{n}$ - number of participants; $\mathrm{fBG}$ - fasting blood glucose; hsCRP - high-sensitivity C-reactive protein.

* $\mathrm{P}<0.05$ OMEGA-3 before vs. after 3 weeks 


\section{Figure captions}

645 Figure 1. The effect of consumption of omega-3 PUFAs enriched (1A) and standard hen 646 eggs (1B) (three eggs per day for three weeks) on post-occlusive reactive hyperemia 647 (PORH) of skin microcirculation in young healthy individuals. Consumption of omega-3 648 PUFAs enriched hen eggs increased skin microvascular PORH in young healthy individuals 649 when compared to PORH measurement before the study protocol (1A). Consumption of standard 650 hen eggs did not induce any significant change in skin microvascular PORH when compared to 651 PORH measurement before the study protocol in young healthy individuals (1B). Results are 652 presented as mean \pm standard deviation (SD). R-O\%- change of microvascular blood flow 653 between reperfusion and occlusion (in relation to baseline). ${ }^{*} \mathrm{P}<0.05$ before vs. after 3 weeks. 
1A: Post-Occlusive Reactive Hyperemia (PORH) in OMEGA-3 group

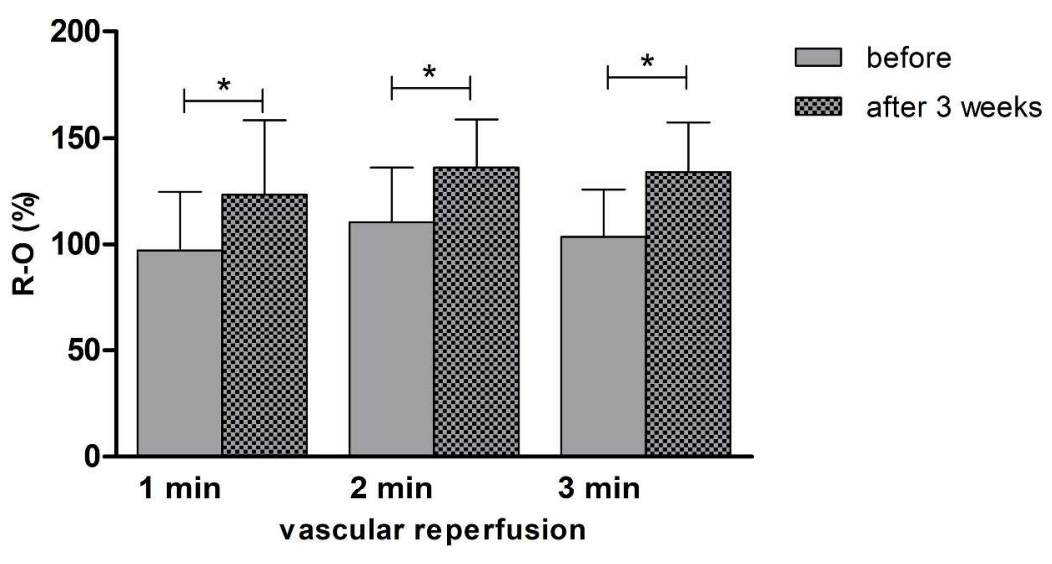

1B: Post-Occlusive Reactive Hyperemia (PORH) in Control group

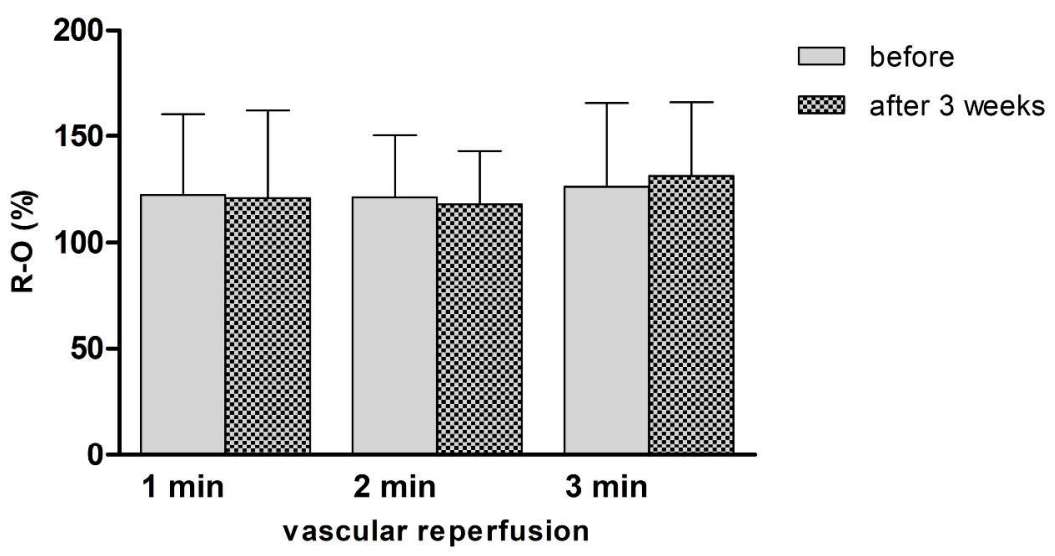

Figure 1

$259 \times 354 \mathrm{~mm}(300 \times 300$ DPI $)$ 